NUC-MN-99/2-T

TPI-MINN-99/10

UMN-TH-99/1746

\title{
Diffractive Structure Function in a Quasi-Classical Approximation
}

\author{
Yuri V. Kovchegov and Larry McLerran \\ School of Physics and Astronomy, University of Minnesota, \\ Minneapolis, MN 55455
}

\begin{abstract}
We derive an expression for diffractive $F_{2}$ structure function which should be valid at small $x$ for quasi-elastic scattering on a hadron and for quasi-elastic scattering on a large nucleus. This expression includes multiple rescatterings of the quark-antiquark pair produced by the virtual photon off the sources of color charge in a quasi-classical approximation. We find that there is a relation between such diffractive production and inclusive processes. In the former, one averages over all colors of sources before squaring the amplitude, and in the latter one first squares the amplitude and then averages it in the hadron's or nuclear wave function. We show that in the limit of a large virtuality of the photon $Q^{2}$ the diffractive structure function becomes linearly proportional to the gluon distribution of the hadron or nucleus, therefore proving that in this sense diffraction is a leading twist effect.
\end{abstract}

\section{INTRODUCTION}

In recent papers by Buchmüller, Hebecker and collaborators [1 -3] diffractive processes are considered where a virtual photon scatters on a hadron or a nucleus, producing two jets out of a quark and an antiquark in the virtual photon's wave function, and a rapidity gap between the quark-antiquark pair and the hadron (nucleus) with which the virtual photon scatters. In the final state the hadron (nucleus) forms a cluster of diffractively produced soft particles. The process is illustrated in Fig. 17a. Buchmüller, Hebecker and colleagues [1 3 ] make the provocative claim that such processes may be computed in a semi-classical description of small $x$ processes where the effect of the hadron is taken into account as classical sources of color charge [4 10 . They claim that such diffractive processes may be computed by first computing the amplitude of the process, then averaging it over the various color orientations of the source and finally squaring the amplitude to obtain the cross section. That way the squaring of the amplitude and averaging in the hadron's (nuclear) wave function are done in the order opposite to what one usually does when calculating total (inclusive) cross section.

In this same theory [1] [3], the structure functions for deep inelastic scattering are computed using the same distribution of classical sources. The only difference is that the amplitude is first squared and then averaged over color. If these prescriptions are true, then there is a subtle and deep relation between the structure functions computed in deep inelastic scattering and diffractive virtual photon production of jets.

In this paper, we find that we can derive this relation for a restricted class of diffractive processes with a rapidity gap where the hadron encountered remains intact (see Fig. 11b). We shall call such processes quasielastic virtual photoproduction. Although it might be possible to extend our methods to the more general case where the hadron fragments into soft particles, we are unable to do so with our current technique.

We shall present two types of arguments. The first is an intuitive argument similar to that invoked in Glauber scattering. We shall pursue the argument in the context of a large nucleus where one has a simple picture of the sources of color charge arising from the nucleon valence quark distribution. We will then later generalize the argument to a hadron where the sources are largely gluonic and arise from a proper renormalization group treatment of the hadronic wavefunction. 
We shall perform this analysis when the averaging over color sources is Gaussian in the same sense as was discussed in [4] 10], or more generally local in transverse space. If the renormalization group analysis after including the QCD evolution in rapidity [8 10] yields the weight function for averaging over sources which is not local, as it well might be, then our prescription would fail to impose the condition of a diffractive rapidity gap on the process at hand and should be understood in the sense of quasi-classical approximation only.

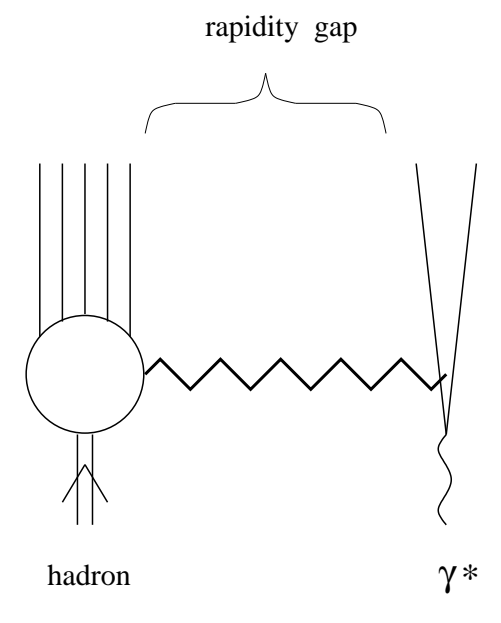

A

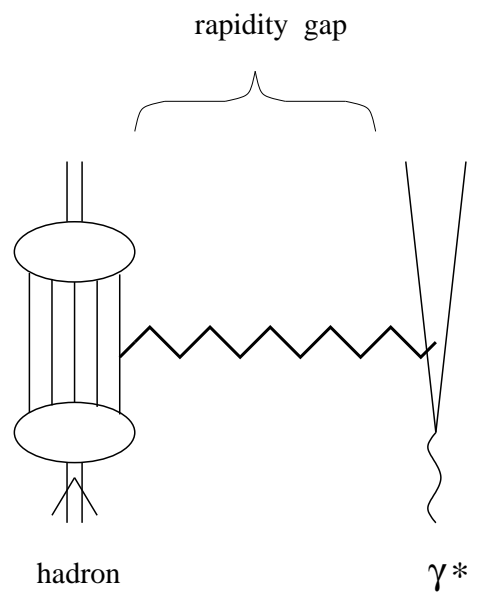

B

FIG. 1. (A) Diffractive deep inelastic scattering with a rapidity gap. (B) The same process with the hadron remaining intact in the final state (quasi-elastic photoproduction). The interaction is roughly illustrated by an exchange of some color singlet particle (pomeron).

We derive explicit expressions for the quasi-elastic structure function of a nucleus or a hadron. This structure function is analogous of the diffractive structure function of Buchmüller, Hebecker and colleagues. We shall refer to it as $F_{2}^{D}$ with the caveat that we are capable at present of describing those processes which are quasi-elastic for the hadron. We also derive an expression for the structure function for deep inelastic scattering $F_{2}$, showing that $F_{2}$ and $F_{2}^{D}$ are related by an interchange of color averaging and squaring of the amplitude.

We analyze the large $Q^{2}$ limit of the expression we obtain for the diffractive structure function $F_{2}^{D}$. The result is very interesting. The diffractive structure function of the hadron or nucleus turns out to be linearly proportional to the gluon distribution of the hadron or nucleus: $F_{2}^{D}\left(x_{B j}, Q^{2}\right) \sim x_{B j} G\left(x_{B j}, Q^{2}\right)$. That way we prove that due to the multiple rescattering effects diffraction becomes a leading twist effect [11], in the sense that diffractive structure function is not suppressed by extra powers of $Q^{2}$ with respect to the total structure function. At large enough $Q^{2}$ diffractive structure function should depend on center of mass energy for deep inelastic scattering, or, equivalently, on Bjorken $x_{B j}$, in the same way as the structure function $F_{2}$. This statement is confirmed by recent HERA data 12.

The outline of the paper is the following: In Sect. II we construct the light cone wavefunction of a virtual photon. This will be useful as we shall derive the scattering amplitude by convoluting this wavefunction with the propagator of the quark-antiquark pair through the hadron which includes multiple scatterings of the $q \bar{q}$ pair on the gluons (classical sources) which constitute the hadron.

In Sect. III we analyze diffractive scattering from a large nucleus and derive an expression for $F_{2}$. This example illustrates all the essential points of the more general analysis where a hadron is treated as a sum of sources arising from a renormalization group treatment of the hadron wavefunction. We show how in the large $Q^{2}$ limit due to the multiple rescattering effects diffractive structure function behaves like a leading twist expression.

In Sect. IV we generalize scattering off a nucleus to a hadron. We argue that if the weight function which is used to color average the sources is Gaussian the result of the previous analysis are essentially reproduced. 
If the averaging procedure is local (but not Gaussian) we shall see how the result above generalizes. We have not been able to generalize to a non-local (in transverse coordinates) averaging procedure.

In Sect. V we summarize our results, and compare with the case of deep inelastic scattering by deriving expressions for the total inclusive cross-section and $F_{2}$ in the quasi-classical limit.

\section{LIGHT CONE WAVE FUNCTION OF A VIRTUAL PHOTON}

Here we are going to calculate the wave function of quark-antiquark fluctuations of a virtual photon. The diagram is shown in Fig. 2. Without any loss of generality we can work in a frame where the transverse momentum of the virtual photon is zero, so that the momentum of the photon is given by $q_{\mu}=\left(q_{+},-\frac{Q^{2}}{2 q_{+}}, \mathbf{0}\right)$ with $Q^{2}$ the virtuality of the photon. The light cone momentum of the virtual photon $q_{+}$is very large, so that in the spirit of the eikonal approximation we will neglect all the inverse powers of $q_{+}$in the calculations below. However we will keep all the inverse powers of $Q^{2}$, therefore resumming all the "higher twist" terms.

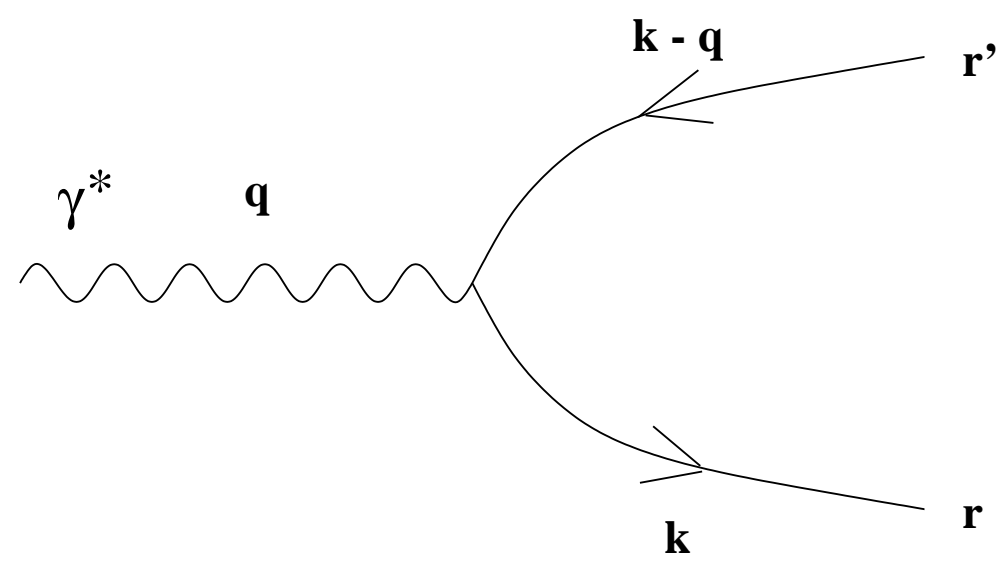

FIG. 2. Light cone wave function of a virtual photon.

Using the light cone perturbation theory with the Feynman rules from 13] one can write down the following expressions for the value of the amplitude in Fig. 2:

$$
\psi_{r r^{\prime}}^{\lambda}(\mathbf{k}, z)=e_{f} \frac{\sqrt{z(1-z)}}{\mathbf{k}^{2}+m_{f}^{2}+Q^{2} z(1-z)} \tilde{u}_{r}(q-k) \epsilon^{(\lambda)} \cdot \gamma v_{r^{\prime}}(k)
$$

with $r$ and $r^{\prime}$ being quark and antiquark helicities correspondingly, $r= \pm 1, \lambda$ is the photon's polarization, $\lambda= \pm 1, m_{f}$ is the quark's mass and $e_{f}$ is its electric charge ( $f$ denotes quark's flavor). We defined $z=k_{+} / q_{+}$as the fraction of the photon's light cone momentum carried by the quark. We will calculate separately the cases of a transverse and longitudinal polarization of the photon. We start with transverse polarization $\epsilon_{T, \mu}^{\lambda}=\left(0,0, \epsilon^{\lambda}\right)$, with $\epsilon^{\lambda}=(1+\lambda i) / \sqrt{2}$. After plugging in the explicit expressions for $\tilde{u}_{r}$ and $v_{r^{\prime}}$ a straightforward calculation yields

$$
\psi_{r r^{\prime}}^{T \lambda}(\mathbf{k}, z)=e_{f} \frac{1}{\mathbf{k}^{2}+m_{f}^{2}+Q^{2} z(1-z)}\left(\delta_{r r^{\prime}} \mathbf{k} \cdot \epsilon^{\lambda}[r(1-2 z)+\lambda]+r \delta_{r,-r^{\prime}} m_{f}(1+r \lambda)\right) .
$$

For the reasons which will become apparent later we want to obtain the virtual photon's wave function in transverse coordinate space. Performing a Fourier transform of Eq. (2)

$$
\psi_{r r^{\prime}}^{\lambda}(\mathbf{x}, z)=\int \frac{d^{2} k}{(2 \pi)^{2}} e^{-i \mathbf{k} \cdot \mathbf{x}} \psi_{r r^{\prime}}^{\lambda}(\mathbf{k}, z)
$$

we obtain 


$$
\psi_{r r^{\prime}}^{T \lambda}(\mathbf{x}, z)=\frac{e_{f}}{2 \pi}\left(\delta_{r r^{\prime}} i \epsilon^{\lambda} \cdot \nabla_{x}[r(1-2 z)+\lambda]+r \delta_{r,-r^{\prime}} m_{f}(1+r \lambda)\right) K_{0}(x a),
$$

where $a^{2}=Q^{2} z(1-z)+m_{f}^{2}$ and $x=|\mathbf{x}|$. Defining

$$
\Phi_{T}(\mathbf{x}, \mathbf{y}, z)=\frac{N_{c}}{2} \sum_{\lambda, r, r^{\prime}, f} \psi_{r r^{\prime}}^{T \lambda}(\mathbf{x}, z) \psi_{r r^{\prime}}^{* T \lambda}(\mathbf{y}, z)
$$

we derive

$$
\Phi_{T}(\mathbf{x}, \mathbf{y}, z)=2 N_{c} \sum_{f} \frac{\alpha_{E M}^{f}}{\pi}\left(a^{2} \frac{\mathbf{x} \cdot \mathbf{y}}{x y} K_{1}(x a) K_{1}(y a)\left[z^{2}+(1-z)^{2}\right]+m_{f}^{2} K_{0}(x a) K_{0}(y a)\right) .
$$

When $\mathbf{x}=\mathbf{y}$ the object in Eq. (6) becomes just a square of the transverse virtual photon's wave function.

To calculate the longitudinal contribution to the wave function we write down an expression for the longitudinal polarization by requiring that $\epsilon^{2}=1$ and $\epsilon \cdot q=0$. We end up with $\epsilon_{L, \mu}^{\lambda}=\left(\frac{q_{+}}{Q}, \frac{Q}{2 q_{+}}, \mathbf{0}\right)$. Similarly to the above one obtains

$$
\psi_{r r^{\prime}}^{L}(\mathbf{k}, z)=e_{f} \frac{1}{\mathbf{k}^{2}+m_{f}^{2}+Q^{2} z(1-z)} 2 Q \delta_{r r^{\prime}} z(1-z) .
$$

Fourier transformation of Eq. (7) yields

$$
\psi_{r r^{\prime}}^{L}(\mathbf{x}, z)=\frac{e_{f}}{2 \pi} \delta_{r r^{\prime}} z(1-z) 2 Q K_{0}(x a),
$$

which results in

$$
\Phi_{L}(\mathbf{x}, \mathbf{y}, z)=2 N_{c} \sum_{f} \frac{\alpha_{E M}^{f}}{\pi}\left[4 Q^{2} z^{2}(1-z)^{2} K_{0}(x a) K_{0}(y a)\right] .
$$

Eqs. (6) and (9) provide us with the square of the virtual photon's wave function:

$$
\Phi(\mathbf{x}, \mathbf{y}, z)=\Phi_{T}(\mathbf{x}, \mathbf{y}, z)+\Phi_{L}(\mathbf{x}, \mathbf{y}, z) .
$$

\section{DIFFRACTIVE STRUCTURE FUNCTION}

In the calculation of the quark-antiquark propagator through the nucleus we will make use of the quasiclassical approximation [15.,16], which restricts the interactions of the $q \bar{q}$ pair with the nucleons to nothing more than a two gluon exchange with the nucleon in covariant gauge. Light cone $\left(A_{-}=0\right)$ gauge analysis of the process is a bit more saddle, but leads to the same formulation of the classical limit [6].

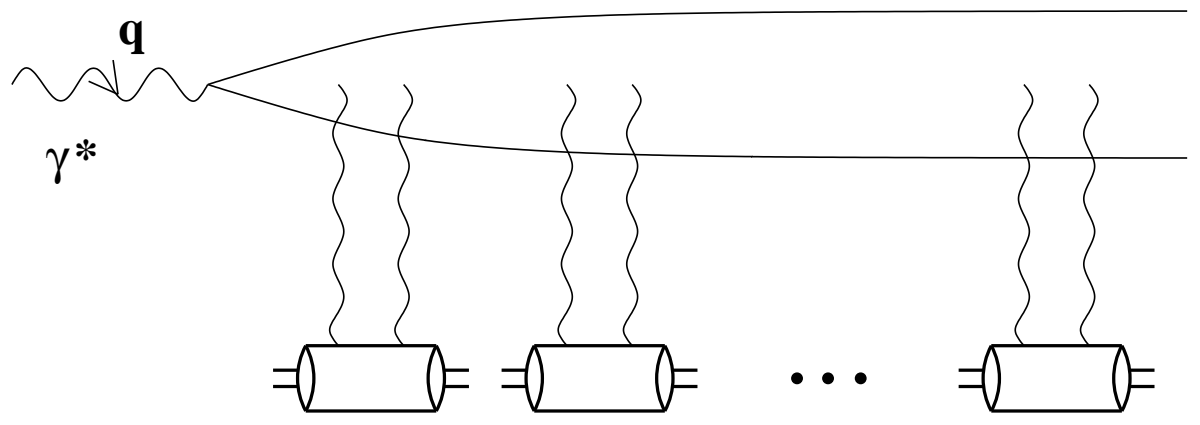

FIG. 3. Diffractive quark-antiquark production in DIS. 
As was stated above, in order to calculate diffractive (or elastic) production of the quark-antiquark pair one has to average the production amplitude in the nuclear wave function. After one does that all the one gluon exchange contributions would cancel resulting in two gluon exchange (virtual) interactions being the only possibility. The amplitude is depicted in Fig. 3. There the gluon lines can hook in all possible ways to the quark lines (see Fig. 4 ). Similarly to the wave functions, the propagator is calculated in the eikonal approximation, i.e., the $q \bar{q}$ pair is assumed to have a very large light cone momentum, inverse powers of which in the amplitude will be neglected.

In order to calculate the quark-antiquark propagator we have to start at the lowest order case of one nucleon by summing the diagrams shown in Fig. 1 . The vertical dashed line there indicates the final state of the $q \bar{q}$ pair. Because the color averaging is done in the amplitude and the complex conjugate amplitude independently, the propagators in the amplitude and the complex conjugate amplitude effectively factorize. That way we can do the summation just in the amplitude and the answer for the complex conjugate amplitude will be the same. That is why in Fig. 0 we show the lowest order interactions only in the amplitude.

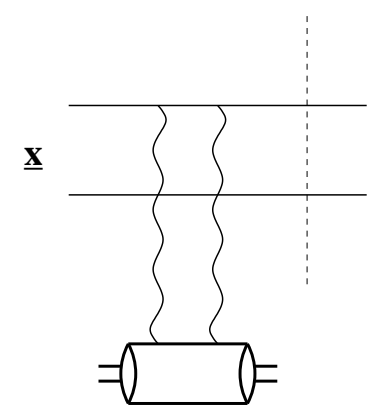

A

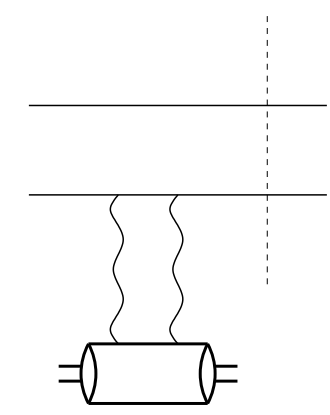

B

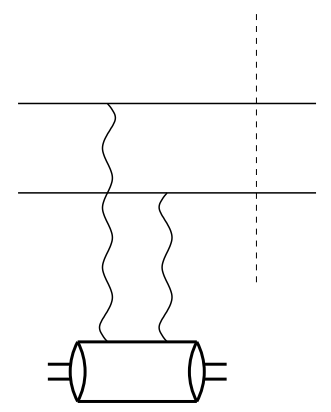

C

FIG. 4. Calculation of the quark-antiquark propagator.

To evaluate diagrams in Fig. [4, similarly to 15, 16, we start by defining the normalized quark-nucleon scattering cross-section

$$
V(\mathbf{l})=\frac{1}{\sigma} \frac{d \sigma}{d^{2}}
$$

where $\mathbf{l}$ is the momentum transfer. The Fourier transformation of $V(\mathbf{l})$ is defined by

$$
\tilde{V}(\mathbf{x})=\int d^{2} l e^{-i \mathbf{l} \cdot \mathbf{x}} V(\mathbf{l}) .
$$

Then, as one can easily see, the diagrams in Fig. 0 give the following factors: A and B give $-(1 / 2) \tilde{V}(\mathbf{0})$ each, and $\mathrm{C}$ gives $\tilde{V}(\mathbf{x})$, where $\mathbf{x}$ is the transverse separation of the pair. Defining

$$
\tilde{v}(\mathbf{x})=\frac{4}{x^{2}}[1-\tilde{V}(\mathbf{x})]
$$

and adding all the contributions of graphs in Fig. 6 we obtain

$$
A+B+C=-[\tilde{V}(\mathbf{0})-\tilde{V}(\mathbf{x})]=-\frac{1}{4} \mathbf{x}^{2} \tilde{v}(\mathbf{x}) .
$$

Now to obtain a contribution to the propagator we have to multiply this result by $\rho \sigma=1 / \lambda$, to take into account the density of nucleons and quark-nucleon cross-section, and by the length of the path along the $z$ direction of the quark-antiquark pair in the nuclear matter $L . \quad \lambda$ is the mean free path of the pair in the nucleus. One can easily see that if we start including more and more nucleons, interacting with the $q \bar{q}$ pair, the answer for the propagator will be just an exponentiation of the lowest order result. Since in light 
cone perturbation theory one has to also include the diagram without interactions, the final answer for the propagator of the pair in the nuclear matter will be

$$
\exp \left(-\frac{\mathbf{x}^{2} \tilde{v}(\mathbf{x}) L}{4 \lambda}\right) .
$$

One should note here that our convention is different from [15], since $\sigma$ in our case is the quark-nucleon cross-section, same as in [16].

An important fact to note is that in the two gluon exchange, no net transverse momentum is carried to the source. The gluon coming in has the same transverse momentum as the gluon going out. Additionally, the longitudinal momentum transfer is very small, of order $e^{-\Delta y}$ where $\Delta y$ is the rapidity gap between the source and the quark-antiquark pair. This rapidity gap will in general be large. Therefore the source barely changes its momentum in the interaction.

In the language of the Fock space wavefunctions one can see that the Fock space wavefunction of the target nucleus is essentially the same after the interaction as it was before. Therefore with probability close to one, the hadronic wavefunction is unchanged. Therefore the procedure of averaging over the sources before squaring the amplitude projects out the quasi-elastic piece of the interaction. In the final state there is a quark-antiquark jet, a large rapidity gap, and finally a nucleus with its momentum largely unchanged.

Of course we expect that a real nucleus having small binding energies will fragment. Presumably quasielastic means here only no inelastic particle production in the central rapidity region. On the other hand for a real hadron, where we imagine the sources as gluons arising from a proper renormalization group treatment of the hadron wavefunction, quasi-elastic means no fragmentation of the hadron.

This leaves open the question of how one treats truly diffractive processes where one allows for some fragmentation of the hadron into pions which have momentum close to the hadron's initial momentum. Presumably one can find a relationship to the quasi-elastic calculation, but we have not been able to do so.

In order to write down the final answer for the $q \bar{q}$ production cross-section it is convenient to work in the rest frame of the nucleus. Analogous to [15] we assume that the virtual photon (or the quark-antiquark pair) reaches the nucleus at light cone time $\tau=x_{-}=0$. Then, if we define $\tau_{1}$ as the time when the virtual photon splits into the quark-antiquark pair in the amplitude and $\tau_{2}$ as the time when the virtual photon splits into the quark-antiquark pair in the complex conjugate amplitude, one should consider four cases:

$$
\begin{array}{ll}
\text { (a) } & \tau_{1}>0, \tau_{2}>0 \\
\text { (b) } & \tau_{1}<0, \tau_{2}>0 \\
\text { (c) } & \tau_{1}>0, \tau_{2}<0 \\
\text { (d) } & \tau_{1}<0, \tau_{2}<0
\end{array}
$$

The corresponding cross-sections will be

$$
\begin{gathered}
\frac{d \sigma^{(a)}}{d^{2} k d z}=\frac{1}{\pi(2 \pi)^{3}} \int d^{2} b d^{2} x d^{2} y e^{-i \mathbf{k} \cdot(\mathbf{x}-\mathbf{y})} \Phi(\mathbf{x}, \mathbf{y}, z) \\
\frac{d \sigma^{(b)}}{d^{2} k d z}=-\frac{1}{\pi(2 \pi)^{3}} \int d^{2} b d^{2} x d^{2} y e^{-i \mathbf{k} \cdot(\mathbf{x}-\mathbf{y})} \Phi(\mathbf{x}, \mathbf{y}, z) \exp \left(-\frac{\mathbf{x}^{2} \tilde{v}(\mathbf{x}) \sqrt{R^{2}-b^{2}}}{2 \lambda}\right) \\
\frac{d \sigma^{(c)}}{d^{2} k d z}=-\frac{1}{\pi(2 \pi)^{3}} \int d^{2} b d^{2} x d^{2} y e^{-i \mathbf{k} \cdot(\mathbf{x}-\mathbf{y})} \Phi(\mathbf{x}, \mathbf{y}, z) \exp \left(-\frac{\mathbf{y}^{2} \tilde{v}(\mathbf{y}) \sqrt{R^{2}-b^{2}}}{\lambda}\right) \\
\frac{d \sigma^{(d)}}{d^{2} k d z}=\frac{1}{\pi(2 \pi)^{3}} \int d^{2} b d^{2} x d^{2} y e^{-i \mathbf{k} \cdot(\mathbf{x}-\mathbf{y})} \Phi(\mathbf{x}, \mathbf{y}, z) \exp \left(-\frac{\mathbf{x}^{2} \tilde{v}(\mathbf{x}) \sqrt{R^{2}-b^{2}}}{2 \lambda}\right) \exp \left(-\frac{\mathbf{y}^{2} \tilde{v}(\mathbf{y}) \sqrt{R^{2}-b^{2}}}{2 \lambda}\right)
\end{gathered}
$$


with $\Phi(\mathbf{x}, \mathbf{y}, z)$ given by Eq. (10). Here $R$ is the radius of the nucleus, which we assume to be spherical, and $b$ is the impact parameter. Adding together the contributions given by Eqs. (17) we find the expression for the quark-antiquark production cross-section in deep inelastic scattering on a nucleus:

$$
\begin{gathered}
\frac{d \sigma^{D}}{d^{2} k d z}=\frac{1}{\pi(2 \pi)^{3}} \int d^{2} b d^{2} x d^{2} y e^{-i \mathbf{k} \cdot(\mathbf{x}-\mathbf{y})} \Phi(\mathbf{x}, \mathbf{y}, z) \\
\times\left[1-\exp \left(-\frac{\mathbf{x}^{2} \tilde{v}(\mathbf{x}) \sqrt{R^{2}-b^{2}}}{2 \lambda}\right)\right]\left[1-\exp \left(-\frac{\mathbf{y}^{2} \tilde{v}(\mathbf{y}) \sqrt{R^{2}-b^{2}}}{2 \lambda}\right)\right] .
\end{gathered}
$$

Making use of the definition of the $F_{2}$ structure function

$$
F_{2}=\frac{Q^{2}}{4 \pi^{2} \alpha_{E M}}\left(\sigma_{T}+\sigma_{L}\right)
$$

together with Eqs. (6), (9),(10), we derive the following formula for the diffractive structure function

$$
\begin{gathered}
F_{2}^{D}=Q^{2} \frac{N_{c}}{\pi(2 \pi)^{3}} \sum_{f} Z_{f}^{2} \int d^{2} b d^{2} x \int_{0}^{1} d z\left\{a^{2} K_{1}^{2}(x a)\left[z^{2}+(1-z)^{2}\right]+m_{f}^{2} K_{0}^{2}(x a)+4 Q^{2} z^{2}(1-z)^{2} K_{0}^{2}(x a)\right\} \\
\times\left[1-\exp \left(-\frac{\mathbf{x}^{2} \tilde{v}(\mathbf{x}) \sqrt{R^{2}-b^{2}}}{2 \lambda}\right)\right]^{2}
\end{gathered}
$$

where $Z_{f}^{2}=\alpha_{E M}^{f} / \alpha_{E M}$ for each quark's flavor. Here we should note that in the logarithmic approximation of small x (large $\left.Q^{2}\right)$

$$
\frac{\tilde{v}(\mathbf{x})}{\lambda}=\frac{2 \pi^{2} \alpha_{s}}{N_{c}} \rho x_{B j} G\left(x_{B j}, 1 / \mathbf{x}^{2}\right)
$$

(see Eq. (4.7) of [16] as well as [19]). Now $\alpha_{s}$ is the strong coupling constant, $\rho$ is the density of nuclear matter, normalized to give the atomic number $A$ when integrated over the whole nucleus. $x_{B j} G\left(x_{B j}, Q^{2}\right)$ is the gluon distribution in a nucleon, which we take at the lowest order in $\alpha_{s}$ (see the next section). Using Eq. (21) one can see now that in the large $Q^{2}$ limit the differential cross section of Eq. (18) becomes proportional to the square of the gluon distribution function of the hadron or nucleus, reducing to an old and well known result [20].

However, the large $Q^{2}$ limit of $F_{2}^{D}$ is more interesting. Let us assume for simplicity of the calculations (only in this section) that our target is a large cylindrical nucleus with the radius $R$ and the length $2 R$ along the axis. Then the impact parameter integration in Eq.(20) becomes trivial giving just a pre-factor of $\pi R^{2}$. Using the representation of the modified Bessel function $K_{1}(\xi)$ 用

$$
K_{1}(\xi)=\frac{\xi}{4} \int_{0}^{\infty} \frac{d t}{t^{2}} e^{-t-\frac{\xi^{2}}{4 t}}
$$

in Eq. (20), assuming that the dominating values of $z$ are small, performing the $z$ and $t$ integrations, we obtain

$$
F_{2}^{D}=\frac{N_{c} R^{2}}{3 \pi^{2}} \sum_{f} Z_{f}^{2} \int_{1 / Q^{2}}^{\infty} \frac{d x^{2}}{\left(x^{2}\right)^{2}}\left[1-\exp \left(-\frac{\alpha_{s} \pi}{2 N_{c} R^{2}} x^{2} x_{B j} G\left(x_{B j}, 1 / x^{2}\right)\right)\right]^{2}
$$

\footnotetext{
${ }^{\dagger}$ We thank D. Kharzeev for showing us this trick.
} 
where $x_{B j} G\left(x_{B j}, Q^{2}\right)$ is now the gluon distribution function of the whole nucleus. In the leading logarithmic approximation we assume that the gluon distribution is a slowly varying function of $Q^{2}$. That way it will effectively play the role of the upper cutoff of the $x$ integration in Eq. (23). Performing the integration in Eq. (23) we obtain the following expression for the diffractive structure function at large $Q^{2}$ :

$$
F_{2}^{D}\left(x_{B j}, Q^{2}\right)=\frac{\alpha_{s}}{6 \pi} \sum_{f} Z_{f}^{2} x_{B j} G\left(x_{B j}, Q^{2}\right) .
$$

A little more careful estimates would introduce a factor of $2 \ln 2$ in front of $x_{B j} G$ on the right hand side of (24). One should note here that the exact coefficient can not be precisely fixed by this method, since to be able to use $x_{B j} G$ as a cutoff in Eq. (23) one should reach the kinematic region of large gluon distributions. This would correspond to evolving $x_{B j} G\left(x_{B j}, Q^{2}\right)$ with some QCD evolution. However, in the strict sense, our $x_{B j} G$ should be taken only at the lowest order in $\alpha_{s}$ [see Eq. (31) below]. Nevertheless our qualitative conclusions should not be altered by introducing evolution in the gluon distributions.

That way we see that the diffractive structure function in the large $Q^{2}$ limit is linear in $x_{B j} G$ with no $Q^{2}$ suppression. Due to the multiple rescattering effects, the $F_{2}^{D}$ structure function becomes a leading twist expression, i.e., it is not suppressed by powers of $Q^{2}$. By resumming higher twist terms we obtained an answer which is effectively leading twist. This result agrees with the previous predictions of [11,21]. Eq. (24) shows that the diffractive structure function depends on energy in the same way as the structure function $F_{2}$ at large $Q^{2}$. This result agrees with the recent ZEUS data [12].

\section{GENERALIZING TO HADRONS}

To generalize the considerations above to the case of a hadron, we first recognize that the renormalization group treatment proposed in 8 10] replaces the gluon distribution of a hadron by a set of sources which include both valence quarks and sea gluons. If we go to small enough $\mathrm{x}$, the gluon density per unit area is large enough so that one can use a weak coupling analysis. In this respect, the situation is entirely parallel to that for a nucleus.

The essential difference is that the function which we use to average over sources changes. If we use a Gaussian distribution of sources,

$$
\int[d \rho] \exp \left(-\int d^{2} \mathbf{x} \frac{\rho^{2}(\mathbf{x})}{2 \Lambda(y)}\right)
$$

then it is an easy exercise to show that the result of the last section are reproduced. To do this we use the fact that the factor $\Lambda(y)$ is the charge squared per unit area [as defined in [5]] with $y \sim \ln x_{+}$the space-time rapidity, and the DGLAP equations [22]. As was discussed in some detail by McLerran and Venugopalan in [5] the propagator of a single quark through the nucleus can be represented as a path ordered exponential (Wilson line)

$$
P \exp \left(i \int_{x_{+}}^{\infty} d x_{+}^{\prime} A_{-}\left(x_{+}^{\prime}\right)\right),
$$

where the field $A_{-}$is the gluon field taken in covariant gauge. Direct calculation of the path ordered integral done in [5] yields for the propagator of the quark-antiquark pair through the hadron [see formula (119) in [5]

$$
\gamma(\mathbf{x})=\exp \left[\alpha^{2} \pi C_{F} \mathbf{x}^{2} \ln \left(\mathbf{x}^{2} \Lambda_{Q C D}^{2}\right) A \Lambda(y)\right],
$$

where the no interaction contribution was included in the propagator in Eq. (27). $A$ is the atomic number of the nucleus. Using the fact that

$$
A \Lambda(y)=\frac{\sqrt{R^{2}-b^{2}} \rho}{C_{F}} \int_{x_{B j}}^{1} d x^{\prime} G\left(x^{\prime}, 1 / \mathbf{x}^{2}\right)
$$


with $\rho=\frac{A}{(4 / 3) \pi R^{3}}$ the nucleon number density in the nucleus we obtain

$$
\gamma(\mathbf{x})=\exp \left(\alpha^{2} \pi \sqrt{R^{2}-b^{2}} \mathbf{x}^{2} \ln \left(\mathbf{x}^{2} \Lambda_{Q C D}^{2}\right) \rho \int_{x_{B j}}^{1} d x^{\prime} G\left(x^{\prime}, 1 / \mathbf{x}^{2}\right)\right),
$$

To establish the correspondence between the result of Eq. (29) and the $q \bar{q}$ propagators from Sect. III one should make use of the double logarithmic limit of the DGLAP equation

$$
\frac{\partial}{\partial \ln \left(Q^{2} / \Lambda_{Q C D}^{2}\right)} x_{B j} G\left(x_{B j}, Q^{2}\right)=\frac{\alpha N_{c}}{\pi} \int_{x_{B j}}^{1} d x^{\prime} G\left(x^{\prime}, Q^{2}\right) .
$$

In the quasi-classical calculation of the previous section the gluon distribution function were taken only at the two gluon level. Noting that in that two gluon exchange approximation the gluon distribution is given by

$$
x_{B j} G\left(x_{B j}, Q^{2}\right)=\frac{\alpha C_{F}}{\pi} \ln \frac{Q^{2}}{\Lambda_{Q C D}^{2}}
$$

one can easily see that Eq. (29) reduces to

$$
\gamma(\mathbf{x})=\exp \left(-\frac{\alpha \pi^{2} \sqrt{R^{2}-b^{2}}}{N_{c}} \mathbf{x}^{2} \rho x_{B j} G\left(x_{B j}, 1 / \mathbf{x}^{2}\right)\right),
$$

which matches the exponents employed in derivation of Eqs. (18) and (20) if one substitutes Eq. (21) into them. That way we showed that the result derived for the structure function of a nucleus [Eq. (20)] is, probably, also valid for a hadron.

There are two assumptions here. First is that one can use the DGLAP equations and the second is that the distribution of sources is local and Gaussian. These are true when one is at high enough momentum transfer so that the density of gluon $d N / d^{2} p_{T} d y$ per unit area is small. This distribution at large $p_{T}$ goes as $1 / p_{T}^{2}$. As the transverse momentum is lowered therefore the density becomes large and our assumptions break down.

The greatest complication in the small $p_{T}$ region $\left(p_{T}^{2}\right.$ less than or of the order of the color charge density of gluon per unit area) is that the Gaussian distribution changes to a presumably non-Gaussian and non-local (in transverse space) form. In the exchanges of gluons between the produced jet of quark-antiquark pairs, some transverse momentum can be absorbed by the source. For local Gaussian distributions

$$
\left\langle\rho\left(\mathbf{x}_{1}\right) \ldots \rho\left(\mathbf{x}_{N}\right)\right\rangle
$$

factorizes into a product of terms such as

$$
\left\langle\rho\left(\mathbf{x}_{1}\right) \rho\left(\mathbf{x}_{2}\right)\right\rangle \sim \delta^{(2)}\left(\mathbf{x}_{1}-\mathbf{x}_{2}\right) .
$$

The locality of this product and its translational invariance are what guarantee that the source absorbs no momentum.

If there is non-locality in the Gaussian averaging, then one couples together sources at different transverse coordinates. There will be overall no transverse momenta transferred to the sum of sources, but there may be momenta transferred to the individual sources at their respective transverse coordinates. (We have a picture in mind where we have coarse grained in transverse coordinate. Each coordinate cell contains a separate source.) If this is the case, then the Fock space wavefunction of the hadron has been modified somewhat from its original form as in the amplitude one has redistributed the transverse momentum among its various constituents.

The problem has therefore become immensely more complicated. One should note however that this can only affect the soft transverse momentum part of the hadron wavefunction, so that perhaps this does not spoil the wavefunction so much. It may also turn out that the non-localities in the renormalization group improved distribution function for sources is small.

As a practical matter, the transverse momentum scale of interest for the non-linearities is presumably the virtuality of the photon $Q^{2}$. If one is at large $Q^{2}$, then non-linearities and non-locality is not important. In this case, the exponential factors in Eq. (20) linearize, and one is back to the simple two gluon exchange model for diffractive processes. Such a model has been derived in the literature [20] from various considerations different than those above. 


\section{TOTAL INCLUSIVE CROSS SECTION}

In the calculation outlined above we have derived the expressions for diffractive (elastic) quark-antiquark pair production and structure function in DIS on a nucleus in the quasi-classical approximation [Eqs. (18), (20)]. Our results include all higher twist effects at the classical level. As was noted above the classical limit in our case is understood as modeling the interactions by no more than two gluons per nucleon. Quantitatively that results in $\alpha_{s}^{2} A^{1 / 3}$ being the expansion parameter. That way our calculation could be understood as resumming all powers of this parameter.

We should note here that similar results were obtained by Buchmüller, McDermott and Hebecker in [2]. However, our expressions in Eqs. (18), (20) give a more explicit power of the exponential in the propagator of the pair through the nucleus.

We should also note that using the techniques outlined above and used previously in [15] one can also calculate the total (inelastic) $q \bar{q}$ production cross-section. The $q \bar{q}$ propagator calculation is a little more difficult. In the framework of the quasi-classical approach one has to also include one gluon exchanges, which leave the nucleon in the color octet state, therefore breaking it apart. Without giving any details we state the results [18]. The quark-antiquark total production cross-section is

$$
\begin{gathered}
\frac{d \sigma}{d^{2} k d z}=\frac{1}{\pi(2 \pi)^{3}} \int d^{2} b d^{2} x d^{2} y e^{-i \mathbf{k} \cdot(\mathbf{x}-\mathbf{y})} \Phi(\mathbf{x}, \mathbf{y}, z) \\
\times\left[1-\exp \left(-\frac{\mathbf{x}^{2} \tilde{v}(\mathbf{x}) \sqrt{R^{2}-b^{2}}}{2 \lambda}\right)-\exp \left(-\frac{\mathbf{y}^{2} \tilde{v}(\mathbf{y}) \sqrt{R^{2}-b^{2}}}{2 \lambda}\right)\right. \\
\left.+\exp \left(-\frac{(\mathbf{x}-\mathbf{y})^{2} \tilde{v}(\mathbf{x}-\mathbf{y}) \sqrt{R^{2}-b^{2}}}{2 \lambda}\right)\right],
\end{gathered}
$$

where the transverse momentum of one of the quarks is fixed to be $\mathbf{k}$ and the momentum of the other is integrated over. This formula reduces to a similar formula derived in [2] [Eq. (32)] only in the logarithmic limit, when we can neglect the $\mathbf{x}$ dependence in $\tilde{v}(\mathbf{x})$. The nuclear structure function in this quasi-classical limit is

$$
\begin{array}{rl}
F_{2}=Q^{2} \frac{2 N_{c}}{\pi(2 \pi)^{3}} \sum_{f} Z_{f}^{2} \int d^{2} b d^{2} & x \int_{0}^{1} d z\left\{a^{2} K_{1}^{2}(x a)\left[z^{2}+(1-z)^{2}\right]+m_{f}^{2} K_{0}^{2}(x a)+4 Q^{2} z^{2}(1-z)^{2} K_{0}^{2}(x a)\right\} \\
\times & {\left[1-\exp \left(-\frac{\mathbf{x}^{2} \tilde{v}(\mathbf{x}) \sqrt{R^{2}-b^{2}}}{2 \lambda}\right)\right]}
\end{array}
$$

which agrees with the previously derived expressions [see [5.14] and references therein]. In the limit of large $Q^{2}$ Eq. (36) provides us with the usual leading twist result

$$
\frac{\partial F_{2}}{\partial \ln \left(Q^{2} / Q_{0}^{2}\right)}=\frac{\alpha_{s}}{3 \pi} \sum_{f} Z_{f}^{2} x_{B j} G\left(x_{B j}, Q^{2}\right) .
$$

That way we have established a procedure of inclusion of higher twist terms in the nuclear or hadronic structure functions and cross sections in the quasi-classical approximation. In both cases of diffractive and total inclusive cross sections the calculation is simple and follows the same prescription. One has to convolute the wave functions of a virtual photon with the propagator of the quark-antiquark pair through the nucleus. For the case of total inclusive cross section one should first square the propagator and then average it in the nuclear wave function. In the case of diffractive process one has to first average the propagator in the nuclear wave function and then square it. 
The relationship between the expressions (20) and (36) can be shown in a different way [23]. Let us rewrite the total and elastic cross sections for the deep inelastic scattering on a hadron or a nucleus in terms of the $S$-matrix at a given impact parameter of the collision, $S(b)$, as 23 .

$$
\begin{gathered}
\sigma_{t o t}=2 \int d^{2} b[1-S(b)] \\
\sigma_{e l}=\int d^{2} b[1-S(b)]^{2} .
\end{gathered}
$$

Now one can see that the diffractive (quasi-elastic) cross sections of a quark-antiquark pair interacting with the nucleus, which was used in Eq. (20), corresponds to the formula (38b), whereas the total inclusive cross section used in formula (36) corresponds to Eq. (38a). Eqs. (38a) and (38b) show how easily the quasi-elastic and and total cross sections could be obtained from one another.

\section{ACKNOWLEDGMENTS}

The authors would like to thank Genya Levin, Alfred Mueller and Raju Venugopalan for many insightful suggestions and commentaries. We thank the anonymous referee for a particularly perspicacious comment which led us to understanding that $F_{2}^{D}$ is a leading twist effect. We are grateful to Urs Wiedemann for pointing out a mistake in the earlier version of the paper. This work is supported by DOE grant DE-FG0287ER40328.

[1] A. Hebecker, Nucl.Phys. B505, 349 (1997).

[2] W. Buchmüller, M. F. McDermott, and A. Hebecker, Phys. Lett. B 410, 304 (1997).

[3] W. Buchmüller, T. Gehrmann, and A. Hebecker, Nucl.Phys. B537, 477 (1999).

[4] L.McLerran and R. Venugopalan, Phys.Rev.D49 (1994) 2233; 49(1994) 3352; 50 (1994) 2225.

[5] L.McLerran and R. Venugopalan, hep-ph/9809427.

[6] Yu. V. Kovchegov, Phys. Rev. D 545463 (1996); D 555445 (1997).

[7] Yu. V. Kovchegov, Dirk H. Rischke, Phys. Rev. C 561084 (1997).

[8] J. Jalilian-Marian, A. Kovner, L. McLerran, and H. Weigert, Phys. Rev. D 55, 5414 (1997).

[9] J. Jalilian-Marian, A. Kovner, A. Leonidov, and H. Weigert, Nucl. Phys. B 504, 415 (1997); Phys. Rev. D 59 014014 (1999); hep-ph/9807462.

[10] J. Jalilian-Marian, A. Kovner, and H. Weigert, Phys. Rev. D 59014015 (1999).

[11] B. Z. Kopeliovich, Phys. Lett. B 447, 308 (1999), and references therein.

[12] ZEUS Collaboration, J. Breitweg et al, Eur. Phys. J. C6, 43 (1999).

[13] G. P. Lepage and S. J. Brodsky, Phys. Rev. D 22, 2157 (1980).

[14] A.H. Mueller, Nucl. Phys. B335, 115 (1990).

[15] Yu. V. Kovchegov, A.H. Mueller, Nucl. Phys. B 529, 451 (1998).

[16] R. Baier, Yu.L. Dokshitzer, A.H. Mueller, S. Peigne, and D. Schiff, Nucl. Phys. B484, 265 (1997).

[17] N.N. Nikolaev and B.G. Zakharov, Z. Phys. C 49, 607 (1991).

[18] D. Kharzeev, Yu. V. Kovchegov, L. McLerran, and R. Venugopalan, in preparation.

[19] E. M. Levin, Phys. Lett. B380 399 (1996); E.M. Levin and M.G. Ryskin Sov. J. Nucl. Phys. 45150 (1987).

[20] L.V. Gribov, E.M. Levin, M.G. Ryskin, Phys. Rept. 1001 (1983); M. Ryskin, Sov.J. Nucl. Phys. 52 529 (1990); M.Ryskin, Z. Phys. C 5789 (1993); S.J. Brodsky, L. Frankfurt, J. F. Gunion, A. H. Mueller, and M. Strikman, Phys. Rev. D 503134 (1994); E. Levin and M. Wusthoff, Phys. Rev. D 504306 (1994).

[21] W. Buchmüller, A. Hebecker, Nucl. Phys. B476, 203 (1996).

[22] Yu. L. Dokshitzer, JETP 73, 1216 (1977); V. N. Gribov and L. N. Lipatov, Sov. J. Nucl. Phys. 15, 78 (1972); G. Altarelli and G. Parisi, Nucl. Phys. B126, 298 (1977).

[23] A. H. Mueller, hep-ph/9710531. 